\title{
Insilico Prediction of Structure, Physico-chemical Properties, and Antigenicity of Fiber Protein of a Fowl Adenovirus (FAdV-D) Field Isolate
}

\author{
Garima Pandey ${ }^{1}$, Dhruv Desai ${ }^{2}$, Aakanksha Tiwari ${ }^{1}$, \\ Anupama Mishra ${ }^{3}$ and Rajesh Kumar ${ }^{1 *}$ \\ ${ }^{1}$ College of Veterinary \& Animal Sciences, Govind Ballabh Pant University of \\ Agriculture \& Technology, Pantnagar, Uttarakhand, India \\ ${ }^{2}$ Vanbandhu College of Veterinary Science and Animal Husbandry, \\ Navsari Agricultural University, Navsari, Gujarat, India \\ ${ }^{3}$ College of Community Sciences, Central Agricultural University (Imphal), Tura, \\ Meghalaya, India \\ *Corresponding author
}

\section{Keywords}

Fowl adenovirus, Fiber protein,

Vaccine,

Antigenicity,

Physico-chemical properties

Article Info

Accepted:

10 January 2021

Available Online:

10 February 2021
Fowl adenovirus (FAdV) belongs to Adenoviridae family of genus Aviadenovirus and classified in five species (Fowl aviadenovirus $A$ to $E$ ) and 12 serotypes ( 1 to $8 \mathrm{a}$ and $8 \mathrm{~b}$ to 11). FAdVs cause variety of clinical conditions in poultry and other birds. Hexon, penton base and fiber are three major structural viral proteins of fowl adenovirus. Fiber protein inherits the epitopes, which are specific for determination of subgroup and type of FAdV. It also plays role in viral penetration as it interacts with the host cellular receptors, hence it has role in the pathogenicity of FAdVs. Therefore, analysis of the structure and properties of fiber protein is important in vaccine development. Present study describes the analysis of fiber protein to determine its antigenicity, physico-chemical properties, secondary and tertiary structures. Fiber protein sequence was retrieved from GenBank, NCBI. Secondary and tertiary structure of protein was predicted by using Psipred V4.0 and phyre 2 software, respectively. Physical and chemical properties of fiber protein were determined by using ProtParam tool. Antigenicity of fiber protein was determined by using VaxiJen v2.0 server. Total twelve physico-chemical properties of fiber protein were determined and overall antigenicity score of 0.5002 was predicted.

\section{Introduction}

Poultry industry is a growing component in the agriculture sector as India is one of the world's largest producers of eggs and broiler meat (APEDA, 2018). Due to introduction of high-performance breeds and intensive farming practices to maximize production, the poultry industry is witnessing increased incidence of viral, bacterial, parasitic, and nutritional diseases. FAdV causes the number of clinical diseases in domestic chicken, such as Inclusion Body Hepatitis-Hydropericardium Syndrome (IBH-HPS), 
adenoviral gizzard erosion (AGE) and tenosynovitis (Schachner et al., 2017). Fowl adenovirus belongs to the family Adenoviridae of genus Aviadenovirus. Twelve serotypes (FAdV- 1 to $-8 \mathrm{a}$ and FAdV-8b to 11) of FAdV have been identified under five species viz., Fowl aviadenovirus $A$ to $E$ based on a cross-neutralization test (Harrach et al., 2011; Harrach and Kajan, 2011; Hess, 2000). IBH-HPS characterized by the accumulation of straw-colored fluid in the pericardial sac and infiltration of basophilic intra-inclusion bodies in the liver (Li et al., 2017).

The major structural adenoviral proteins are hexon and penton, constituting an icosahedral capsid of 252 capsomeres. Penton is composed of penton base and attached to it that are two fibers of equal length, a peculiarity of fowl adenoviruses (Marek et al., 2012). The penton base anchors the antenna-like fiber protein, whose distal head domain, termed knob, harbors the receptorbinding site thus, essential for initiating virus attachment to the host cell (Wang and Jhao, 2019). All type of epitopes such as type specific neutralizing, subgenus-specific neutralizing, and type specific nonneutralizing epitopes exist within the fiber. The adenoviral fiber protein has been shown to be related to the tissue tropism as it has ability to bind with the cell surface therefore, it has role in the pathogenicity of FAdV (Henry et al., 1994; Pallister et al., 1996; Shah et al., 2012). In the present study, we predict the secondary and tertiary structure, Physico-chemical properties and antigenicity of fiber protein that will be beneficial for vaccine design in future.

\section{Materials and Methods}

\section{Protein sequence retrieval}

Fiber protein sequence of a Fowl adenovirus D isolate Pantnagar/HA-14/R-21 (Accession
No. MH371143) was retrieved from GenBank of National Center for Biotechnology Information (NCBI).

Determination of the secondary and tertiary structure of Fiber protein

Secondary structure of fiber protein was determined by using Psipred V4.0 tool (http://bioinf.cs.ucl.ac.uk/psipred/) and tertiary structure of the protein was identified by using Phyre2 tool (http://www.sbg.bio.ic.ac.uk/phyre2/html/pag e.cgi?id=index).

\section{Determination of physical and chemical parameters of fiber protein}

The retrieved sequence of fiber protein of FAdV was assayed for physical and chemical properties by using the ProtParam tool (https://web.expasy.org/protparam/).

\section{Antigenicity analysis of Fiber protein}

VaxiJen v2.0 server (http://www.ddgpharmfac.net/vaxijen/VaxiJen/VaxiJen.html) was used to determine the antigenicity of fiber protein. VaxiJen v2.0 server uses physicochemical properties of proteins to develop alignment-independent prediction (Doytchinova and Flower, 2007). The threshold value for the selected target organism for the software was 0.4.

\section{Results and Discussion}

\section{Physical and chemical properties of Fiber protein}

Fiber protein of FAdV strain was examined by ProtParam tool for estimating different physical and chemical properties such as molecular weight, theoretical pI, amino acid composition, atomic composition, estimated half-life, instability index, aliphatic index, and 
grand average of hydropathicity (GRAVY). Results of these computed parameters are shown in table 1. Similarly, ProtPram tool was also used to determine the physicochemical properties (such as molecular weight, theoretical pI, amino acid composition, atomic composition, extinction coefficient, estimated half-life, instability index, aliphatic index, and grand average of hydropathicity (GRAVY)of boAP3D1, Bovine Leukemia Virus Envelope (BLV Env) protein and recombinant proteins (Corredor $e t$ al., 2018).

\section{Prediction of Secondary and Tertiary structure of Fiber protein}

The structural prediction was carried out to find out alpha helix and beta pleated sheet and its interaction. Secondary structure prediction through the Psipred V4.0 tool revealed that there are more than twenty strands in protein while most of the amino acids are of membrane interaction and transmembrane helix (Fig. 1). The tool could not predict alpha helix. The tertiary structure was predicted by homology modelling through a database available. A homology model was created to identify similarity prediction. It was made with $100 \%$ confidence and coverage was $35 \%$, which includes 196 residues by the single highest scoring template (Fig. 2). Similarly, Pesipred V4.0 web tool is also used to determine the secondary structure of SARS-CoV-2 structural proteins for multiepitope vaccine development (Kumar et al., 2020). Tertiary structure of envelope glycoprotein of DENV-2 and DENV-3 for the development of dengue vaccine was also predicted through Phyre2 tool (Rasool et al., 2015).

Table.1 Physical and chemical properties of fiber protein of Fowl adenovirus

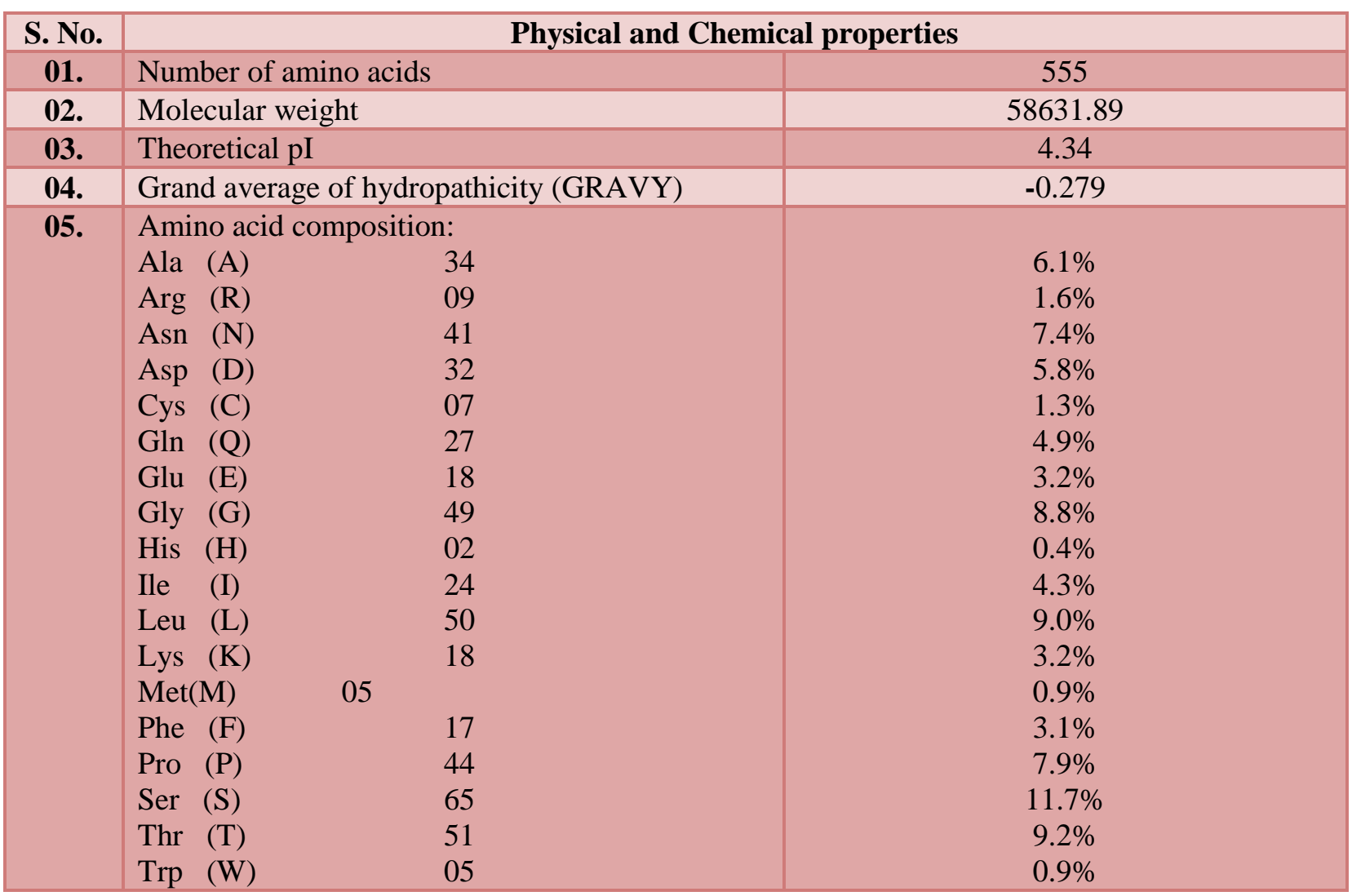




\begin{tabular}{|c|c|c|}
\hline & $\begin{array}{lll}\text { Tyr } & (Y) & 21 \\
\text { Val } & (\mathrm{V}) & 36 \\
\text { Pyl } & (\mathrm{O}) & 00 \\
\text { Sec } & (\mathrm{U}) & 00\end{array}$ & $\begin{array}{l}3.8 \% \\
6.5 \% \\
0.0 \% \\
0.0 \%\end{array}$ \\
\hline 06. & Estimated half life & $\begin{array}{l}30 \text { hours (mammalian reticulocytes, in } \\
\text { vitro) } \\
>20 \text { hours (yeast, in vivo) } \\
>10 \text { hours (Escherichia coli, in vivo) }\end{array}$ \\
\hline 07. & Instability Index & 34.24 \\
\hline 08. & Aliphatic Index & 76.94 \\
\hline 09. & $\begin{array}{ll}\text { Atomic composition: } \\
\text { Carbon } & \text { (C) } \\
\text { Hydrogen } & (\mathrm{H}) \\
\text { Nitrogen } & (\mathrm{N}) \\
\text { Oxygen } & (\mathrm{O}) \\
\text { Sulfur } & (\mathrm{S})\end{array}$ & $\begin{array}{c}2577 \\
4005 \\
677 \\
861 \\
12\end{array}$ \\
\hline 10. & Formula & $\mathrm{C}_{2577} \mathrm{H}_{4005} \mathrm{~N}_{677} \mathrm{O}_{861} \mathrm{~S}_{12}$ \\
\hline 11. & Total number of atoms & 8132 \\
\hline 12. & $\begin{array}{l}\text { Total number of negatively charged residues } \\
\text { (Asp + Glu): } \\
\text { Total number of positively charged residues } \\
\text { (Arg + Lys): }\end{array}$ & $\begin{array}{l}50 \\
27\end{array}$ \\
\hline
\end{tabular}

Fig.1 Secondary structure of fiber protein of fowl adenovirus

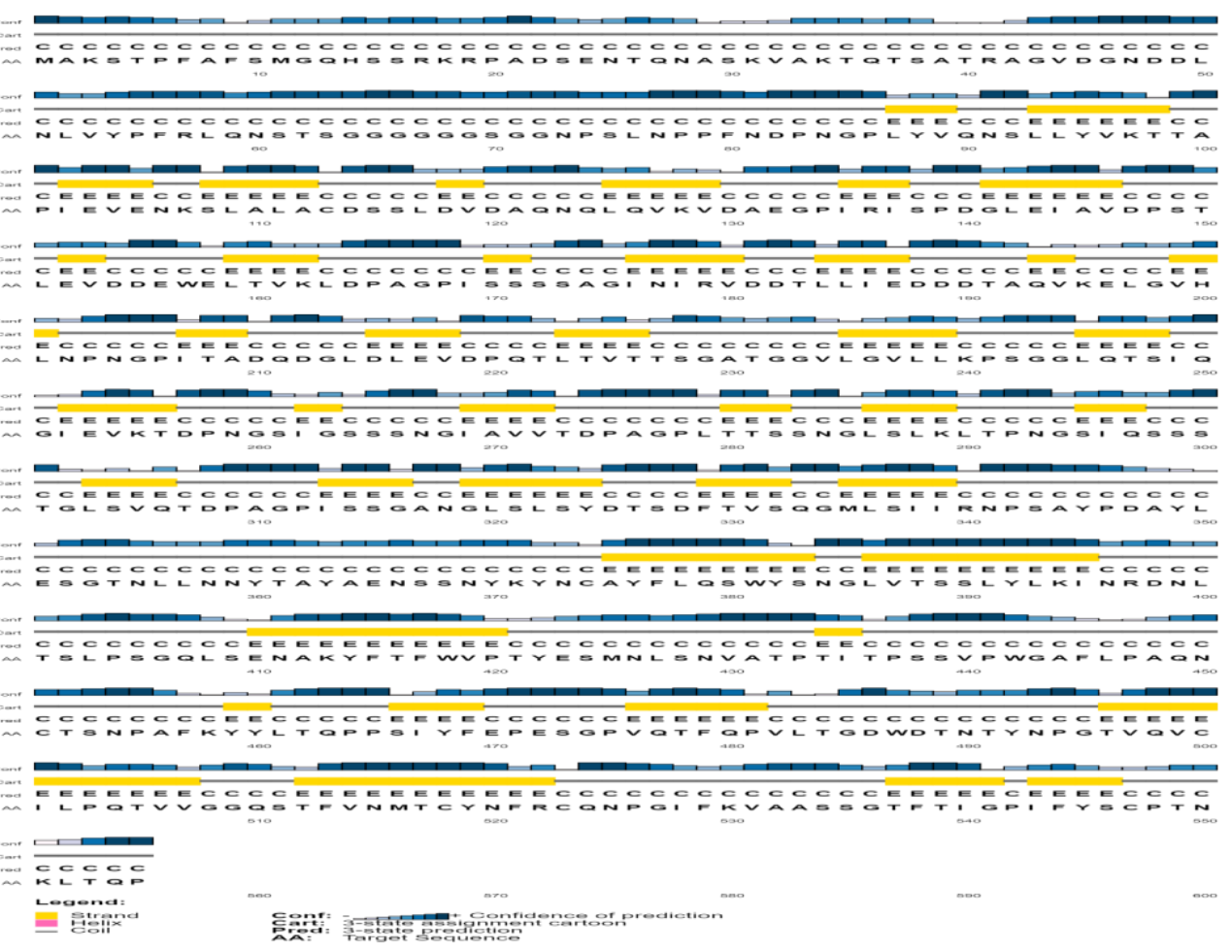


Fig.2 Tertiary structure of fiber protein of fowl adenovirus

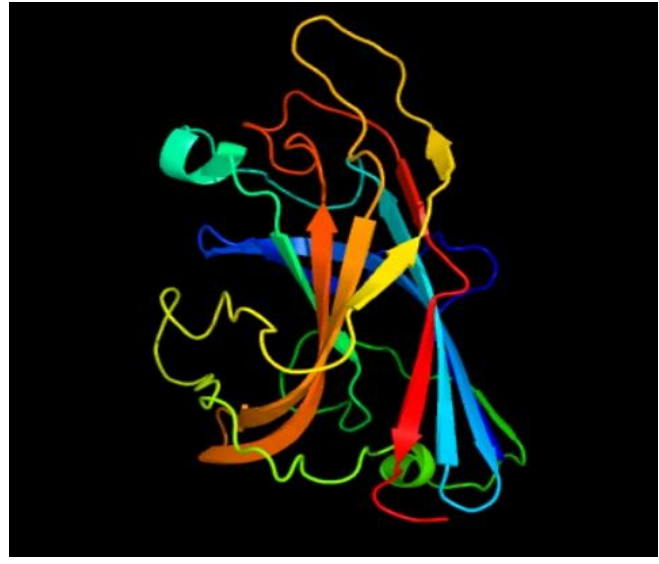

\section{Antigenicity analysis of fiber protein}

The antigenicity of fiber protein of fowl adenovirus was determined by using VaxiJen v2 sever which uses 0.4 as a default threshold value. The server had predicted the overall antigenicity score of 0.5002 . Therefore, fiber protein of fowl adenovirus was found to be probable antigen by VaxiJen v2. Similarly, all sequences of Porcine rubula virus were checked for antigenicity by VaxiJen v2 server. Among all the sequences only the $\mathrm{P}$ protein was not considered as antigen as it has antigenicity score 0.3560 while $\mathrm{HN}$ and $\mathrm{F}$ membrane glycoprotein of Porcine rubula virus were considered as most antigenic proteins $(0.5271$ and 0.5154) (Sianez-Estrad et al., 2020). The VaxiJen server was used in other studies to select promising Human Immunodeficiency Virus (HIV) and Hepatitis $\mathrm{C}$ virus antigens, structural proteins with a VaxiJen score $>0.4$ (Rao et al., 2013; Idrees et al., 2013)

In conclusion three immune informatics tools viz., ProtPram, Psipred V4.0 and VaxiJen v2 were used to predict the physico-chemical properties, secondary and tertiary structure, and antigenicity of most antigenic fiber protein of fowl adenovirus as this protein is capable to induce the production of neutralizing antibodies in the host against the virus. Twelve different physico-chemical properties were predicted by the ProtPram tool. Pesipred V4.0 tool has

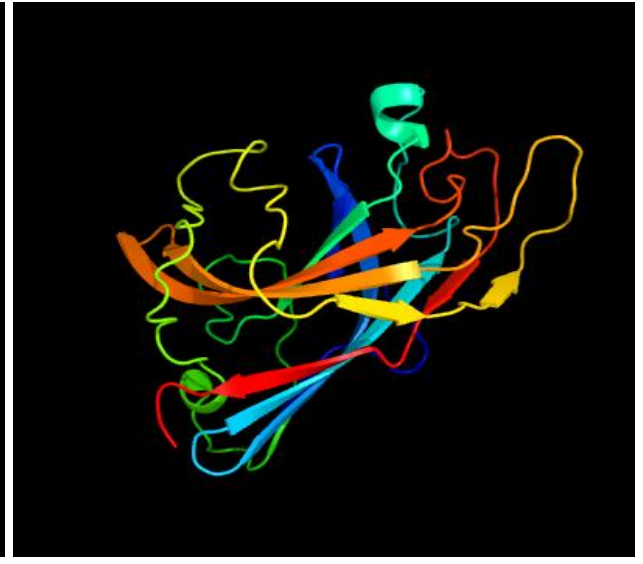

predicted more than twenty strands in protein while most of the amino acids are of membrane interaction and transmembrane helix in secondary structure of fiber protein while in tertiary structure, it was made with $100 \%$ confidence and coverage was $35 \%$, which includes 196 residues by the single highest scoring template. Fiber protein has 0.5002 antigenicity score ( $>4$ by default) as predicted by VaxiJen v2. Therefore, based on the results of different immunoinformatic tools the fiber protein of FAdV is potential antigenic protein and can further be analyzed for the development of peptide vaccine.

\section{Acknowledgement}

Authors are thankful to the Dean College of Veterinary \& Animal Sciences and Director Experiment station, G.B. Pant University of Agriculture \& Technology, Pantnagar for providing necessary facilities to carry out this research work.

\section{References}

Corredor, A.P., González, J., Baquero, L.A., Curtidor, H., Olaya-Galán, N.N., Patarroyo, M.A. and Gutiérrez, M.F. 2018. In silico and in vitro analysis of boAP3d1 protein interaction with bovine leukaemia virus gp51. PLoS one, 13(6), p.e0199397. 
Doytchinova, I.A. and Flower, D.R., 2007. VaxiJen: a server for prediction of protective antigens, tumor antigens and subunit vaccines. BMC bioinformatics, 8(1): 1-7.

Harrach B., and Kaján G.L.2011. The Springer Index of Viruses. 2 ed. Springer-Verlag; New York. Aviadenovirus; pp. 13-28.

Henry, L.J., Xia, D., Wilke, M.E., Deisenhofer, J., and Gerard, R.D.1994. Characterization of the knob domain of the adenovirus type 5 fiber protein expressed in Escherichia coli. Journal of Virology 68 (8), 5239-5246.

Hess, M., 2000. Detection and differentiation of avian adenoviruses: a review. Avian. Pathol. 29 (3), 195-206.

Idrees S, and Ashfaq UA (2013). Structural analysis and epitope prediction of $\mathrm{HCV}$ E1 protein isolated in Pakistan: an insilico approach. Virol J.; 10(1):113.

Khawaja DA., Ahmad S., Rauf MA., Zulfiqar MZ., Mahmood SMI., and Hassan M. 1988. Isolation of an adenovirus from hydropericardium syndrome in broiler chicks. Pak J Vet Res 1:2-17.

Kumar, A., Kumar, P., Saumya, K.U., Kapuganti, S.K., Bhardwaj, T. and Giri, R., 2020. Exploring the SARS-CoV-2 structural proteins for multi-epitope vaccine development: an in-silico approach. Expert review of vaccines, 19(9): 887-898.

Marek, A., Nolte, V., Schachner, A., Berger, E., Schlötterer, C., and Hess, M., 2012. Two fiber genes of nearly equal lengths are a common and distinctive feature of Fowl adenovirus $\mathrm{C}$ members. Veterinary Microbiology 156 (3-4), 411-417.
Pallister, J., Wright, P.J., and Sheppard M. 1996. A single gene encoding the fiber is responsible for variations in virulence in the fowl adenoviruses. Journal of Virology 70 (8), 5115-5122.

Rao BS, Gupta KK, Suchitra K, Gupta A, and PujithaK. 2013. Conserved HIV Wide spectrum antipeptides-A hope for HIV Treatment. Advanced Techniques in Biology \& Medicine. https://doi.org/10.4172/atbm.1000103 PMID: 25077181

Rasool A., H., Saghir, A. and Idrees, M., 2015. Computational prediction and analysis of envelop glycoprotein epitopes of DENV2 and DENV-3 Pakistani isolates: a first step towards Dengue vaccine development. PLoS one, 10(3). p.e0119854.

Schachner A., Matos M., Grafl B., and Hess M. 2017. Fowl adenovirus-induced diseases and strategies for their control - a review on the current global situation. Avian Pathology. 47:1-16.

Shah, M.S., Ashraf, A., Rahman, M., Khan, M.I., and Qureshi, J.A. 2012. A subunit vaccine against hydropericardium syndrome using adenovirus penton capsid protein. Vaccine 30 (50), 7153-7156.

Three Year Export Statement, APEDA. Retrieved from https://agriexchange.apeda. gov.in/indexp/ExportStatement.aspx on 21.05.2020.

Zeng Wang and Jun Zhao. 2019. Pathogenesis of Hypervirulent Fowl Adenovirus Serotype 4: The Contributions of Viral and Host Factors. Viruses (11), 741.

\section{How to cite this article:}

Garima Pandey, Dhruv Desai, Aakanksha Tiwari, Anupama Mishra and Rajesh Kumar. 2021. Insilico Prediction of Structure, Physico-chemical Properties, and Antigenicity of Fiber Protein of a Fowl Adenovirus (FAdV-D) Field Isolate. Int.J.Curr.Microbiol.App.Sci. 10(02): 1396-1401. doi: https://doi.org/10.20546/ijcmas.2021.1002.167 